\title{
Dinámicas y relaciones del emprendimiento desde el ámbito de la psicología
}

Fecha de recepción: 17 de marzo de 2010

Fecha de aprobación: 1 junio de 2010

\author{
José Guillermo Narváez Casallas \\ jnarvaez@unisalle.edu.co \\ Universidad de La Salle \\ Reseña del autor \\ Administrador de empresas, magíster en administración, especialista en Finanzas Privadas. \\ Profesor-investigador de la Universidad de La Salle.
}

\section{Jaime Augusto Porras Jiménez}

jporrasj@gmail.com

Universidad de La Salle

Reseña del autor

Administrador de Empresas, especialista en Administración y Gerencia. Magíster en Administración. Ex ejecutivo, asesor y consultor de empresas del sector servicios, de economía solidaria, de onGS y de mIPYMES. Perito administrativo-financiero de la Superintendencia de Sociedades de Colombia. Coordinador de procesos de autoevaluación y de registro calificado. Profesor universitario en administración, finanzas, economía solidaria, macroeconomía, emprendimiento y técnicas de negociación. Profesor-investigador de la Universidad de La Salle y coordinador de la Unidad de Emprendimiento de la misma institución.

\section{Víctor José Rodríguez Restrepo}

victorj@poligran.edu.co

Politécnico Grancolombiano

\section{Reseña del autor}

Administrador de empresas de la Universidad Externado de Colombia, especialista en Gerencia de Recursos Humanos de la Escuela de Administración de Negocios, EAN; y especialista en Pedagogía Contemporánea de la misma Escuela. Es catedrático de Iniciativa, Desarrollo empresarial y Creación de empresas del Politécnico Grancolombiano.

\section{Resumen}

El emprendimiento desde la psicología, implica examinar importantes aportes, como por ejemplo el de Abraham Maslow (1934), quien plantea que la motivación de emprender está constituida por una

\author{
Abstract \\ Entrepreneurship from the Psychology, involves \\ examining important contributions, such as \\ Abraham Maslow, who says the motivation to \\ undertake consists of a necessity, not a balance \\ or equilibrium includes the person has to do
}


necesidad, que no comprende un balance o equilibrio en la persona; sino que tiene que ver con los continuos deseos del ser, de llenar potencialidades. David McClelland (1972), plantea que "el deseo de éxito, lo lleva a asumir una responsabilidad personal, permitiendo visualizar las metas". Para Howard Stevenson (1990), el problema de emprender depende de la disponibilidad de recursos para llevar a cabo la idea como iniciativa. Gordon Allport (1922), afirma que la conducta del emprendedor es un problema de madurez.

Por otra parte, Carl Rogers (1951), sostiene que el asunto es de personalidad, y que el emprendimiento aparece cuando realmente se tiene conocimiento de sí mismo, se reconocen las necesidades y se ponen en juego sus potencialidades. Para Mitzberg, Ahlstrand y Lampel (1999), el emprendimiento, es la forma como se asocia la estrategia con la visión de negocio, formando un líder personalizado. Para Drucker (1964), la conducta emprendedora implica tomar decisiones con visión e intuición. Holland (2001), determina la personalidad en siete prototipos.

Para Manfred Max Neef (1999), el emprendimiento depende de las necesidades humanas de sobrevivencia las cuales son influenciadas por el entorno en donde se encuentra. Finalmente, para Andy Freire (2005), la razón de emprender es producto del aprendizaje adquirido, se desarrolla habilidades básicas, se prepara en lo técnico, se asume un papel en lo directivo y se enfrenta riesgos para lograr la independencia. El presente texto demuestra los interesantes y profundos aportes de la psicología para entender el emprendimiento, así como la insuficiencia de dicho entendimiento desde tan sólo una disciplina.

\section{Palabras clave}

Emprendimiento, comportamiento, logro, motivación, personalidad. with the continuing desire of be to fill potential. David McClelland (1972), states that "the desire for success, leads him to take personal responsibility, allowing to visualize the goals." For Howard Stevenson (1990), the problem of undertaking depends on the availability of resources to carry out the idea as an initiative. Gordon Allport (1922), says the entrepreneur's behavior is a problem of maturity.

Carl Rogers (1951), argues that the issue is personality, entrepreneurship appears when you really have self-knowledge, recognize their needs, their potential is at stake. To Mitzberg, Ahlstrand and Lampel (1999) entrepreneurship, is how the strategy is associated with business vision, creating a personalized leader. For Drucker (1964), the conduct involves making entrepreneurial vision and intuition. Holland (2001), determines personality in seven prototypes.

For Manfred Max Neef (1999), the venture depends on the human needs of survival, influenced by the environment where you are. Finally, to Andy Freire (2005), the reason to launch is the result of acquired learning, develop basic skills, is prepared in the technical, assumes a role as manager, faces risks to achieve independence. This article shows the interesting and profound contribution of psychology to understand the venture, and the inadequacy of this understanding from just one discipline.

\section{Keywords}

Entrepreneurship, Behavior, Achievement, Motivation, Personality. 


\section{Introducción}

Una persona emprendedora debe estar en capacidad de entender y atender el cambio tecnológico del mundo actual, de reconocer que las sociedades del universo están sufriendo transformaciones culturales, económicas, de conocimiento y de innovación, que se está evolucionando de una sociedad industrial, pasando por una sociedad de la información, para continuar con la del conocimiento. Se está gestando una nueva revolución que afecta el emprendimiento global, en términos de su desarrollo, tamaño, poder de negociación, formas de direccionar la empresa, diversidad étnica, niveles de asociatividad y redes de comunicación. Un emprendedor debe reconocer la importancia de proyectar un plan de vida y de carrera frente a esta situación.

El estudio de la psicología tiene un papel fundamental en el tratamiento de las personas, porque los problemas de actitud y aprendizaje frente al trabajo, han llevado a los estudiosos a predecir comportamientos, maneras de apasionarse $o$ aburrirse por lo que se hace $y$ reconocer sentimientos de autoestima frente a logros o fracasos por proyectos realizados, hasta concebir el tema del emprendimiento como un problema de la personalidad.

Para tratar este tema, se estudian los supuestos expuestos a través de los años, en teorías relacionadas con el emprendimiento y la personalidad, para sentar postulados y premisas acerca de cómo algunas personas, emprenden y otras no, debido a los problemas de carácter psicológico, sobre los que muchos no tienen la posibilidad de incursionar con acierto y autenticidad en el tema. Primero, se hablará de los autores que argumentan que el emprendimiento es un problema de motivación, posteriormente, se mencionarán aquellos que toman al comportamiento como el factor importante en las personas que comienzan una idea creativa; se explicará por qué otros ensayistas mencionan que el problema de emprender está en la personalidad de los individuos, también se analizará si el emprendedor nace o se hace, y finalmente, se abordará el tema del problema vocacional de emprender en la juventud, como una parte primordial de la subsistencia en la sociedad.

\section{Antecedentes del enfoque psicológico o humanista sobre el emprendimiento}

Para Lucas (2004), fue Max Weber, en la perspectiva de analizar el cristianismo -catolicismo, luteranismo y calvinismo-y su impacto en el desarrollo del capitalismo, quien asignó especial importancia a la visión de los calvinistas, "para quienes el éxito material logrado mediante el propio trabajo era, no sólo 
digno, sino que constituía una prueba de la gracia de Dios. Así, conductas y hábitos antes considerados despreciables, se convirtieron en el siglo XVIII en deberes profesionales".

Según el mismo autor, en este contexto se abrió pasó un

nuevo papel del individuo en la producción material, apareciendo los economistas franceses como los primeros que le asignaron valor económico fundamental a la iniciativa personal en el mundo de los negocios, y fue según Krause y Thomas (2004), Richard Cantillón, quien hacia el año 1712, acuñara el término entrepreneur, significando "aquel que tiene iniciativa".

(Lucas, 2004)

Ciertamente, como el emprendedor es el centro de la acción emprendedora, "no se podría abordar el tema de la actividad emprendedora sin precisar quién es el individuo que personifica tal acción”, González (2003); por tanto, aparece con importantes aportes y desarrollos al emprendimiento, la escuela psicológica o enfoque humanista.

Por ello, siguiendo la frase "conócete a ti mismo", grabada en letra de piedra hace 2500 años, en el pórtico del templo de Delfos en la antigua Grecia, ante la reflexión de Wompner (2005), genera los dos pilares fundamentales del proceso del espíritu emprendedor con respecto a las personas: que aprendan a conocerse a sí mismas y a tener confianza en sus propias habilidades y capacidades, ya que el espíritu emprendedor es de lejos la fuerza más poderosa que está detrás del desarrollo económico y la movilidad social, según, Silva (1998), citado por Wompner (2005), pues el espíritu emprendedor se genera con el deseo y la capacidad de cambiar una situación propia o ajena, para mejorarla o transformarla en otra más favorable para el individuo y para su entorno social, según Villa (2008), citado por Wompner (2005).

Según Aragón y Severi (2001), en la escuela psicológica o humanística, es altamente aceptado que la actitud, las creencias y los valores determinan el comportamiento humano -lo que uno es-. Las personas se comportan de acuerdo con sus valores, a pesar de las diferentes situaciones. La escuela psicológica, que se enfoca en factores personales, cree que los emprendedores poseen valores únicos y actitudes hacia el trabajo y la vida, y que los emprendedores pueden ser diferenciados de los no emprendedores por sus características personales. Las personas que poseen las mismas características que los emprendedores, tendrán una tendencia alta a alcanzar actos emprendedores, más que aquellos que no poseen estas características (Lachman, 1980).

Según el mismo autor, tres características personales han recibido una atención considerable en este enfoque: 
1. Los valores personales como la honestidad, la responsabilidad y la ética.

2. La propensión a tomar el riesgo.

3. La necesidad del logro.

Esta escuela o enfoque, afirma que los emprendedores no pueden desarrollarse o entrenarse porque son innatas -nace con el individuo-, y que las características del emprendedor son desarrolladas en su infancia, en su relación familiar, y en su relación con la comunidad -se hace a partir de sus relaciones y experiencias-. Siguiendo especialmente la segunda corriente, según Aragón y Severi (2001), la escuela psicológica cree que ciertos valores individuales y necesidades son la precondición necesaria del emprendedor. Dado que esos valores son aprendidos en la niñez y son reafirmados antes de la adultez, las características del emprendedor difícilmente puedan ser inculcadas en universidades o en las escuelas. Entre estas características, destacan la necesidad de realización, la toma de riesgo, la tolerancia a la ambigüedad y la buena conducta.

En esta escuela, además de los valores anteriores, sobresalen los aportes acerca de las motivaciones; por ejemplo, Castillo (1999), afirma que las motivaciones son la base esencial para el emprendiminento, pues varias investigaciones buscan entender este proceso e incorporar los factores asociados, mediante la investigación profunda sobre la empresa y empresario. Los primeros modelos se basaron en la percepción de que el emprendimiento era una característica de la personalidad según McClelland (1961), Brockhaus (1980 y 1982), la existencia de un modelo psicodinámico, según Kets de Vires (1977), asociado a la marginalidad social de acuerdo a Stanworth y Curran (1973) y, Scase y Goffee (1980 y 1982) o las variables personales de acuerdo con el aprendizaje cognitivo social, según Cehl (1985) y Mischels (1973). Las limitaciones de estos modelos generaron otros estudios basados en motivaciones, entre los que se destacan los desarrollados por Cooper (1970 y 1971), Shapero (1985), Martin (1984), Greenberger y Sexton (1988), Gibb y Ritchie (1982) y, Scheinberg y MacMillan (1988).

En unos y otros modelos se destaca la importancia de las razones que impulsaron el comienzo de la empresa. Dos grupos de investigadores trabajan en esta área: los liderados por Ian MacMillan, de Wharton School de la Universidad de Pennsilvania, y por Harold Welsch, de la DePaul University, en Illinois. De la revisión bibliográfica se destaca el modelo de Scheinberg y MacMillan (1988), basado no en las implicaciones psicológicas asociadas a la formación de empresas, sino a la identificación de veintitrés razones por las cuales un grupo de 2.278 emprendedores de catorce países iniciaron sus empresas. Un estudio posterior de los mismos autores, indicó que en once países existen seis factores asociados a la formación de empresas: 
1. La necesidad de la aprobación.

2. La instrumentalización percibida de la riqueza.

3. El grado de sentido comunitario.

4. La necesidad de desarrollo personal.

5. La necesidad de independencia.

6. La necesidad de escapar.

Este estudio, con la participación de un grupo internacional de investigadores, permitió delimitar los factores a cuatro e incluir las razones de beneficios tributarios según las respuestas de los entrevistados. Los cuatro factores identificados fueron los siguientes:

1. La independencia.

2. El reconocimiento.

3. El aprendizaje.

4. Los roles.

Al analizar los modelos establecidos con anterioridad por otros autores, es posible incluir dentro de estas cuatro áreas algunos motivos señalados como relevantes por otros autores. Cabe mencionar que se aceptan como factores de independencia, las percepciones de empuje y atracción que se contraponen: el miedo inminente a la pérdida del empleo o el sustento y el deseo de aprovechar una oportunidad a pesar del riesgo de la pérdida de la fuente de ingreso segura. Amit y Muller (1994), determinando una manera de evaluar emprendedores con fines de inversión llegaron a la conclusión de que las motivaciones podrían tener una base de esperanza o desesperanza, complementando los estudios anteriores de McMillan. Los estudios de las motivaciones permiten enlazar teorías con acción para favorecer la explicación racional de las decisiones tomadas por emprendedores y su impacto en la empresa. Esto forma parte de las nuevas metodologías de enseñanza que se desarrollan para aumentar el éxito de los programas de capacitación en emprendimiento y que se mencionarán a continuación.

\section{La motivación como factor de emprendimiento desde Abraham Maslow (1934)}

La motivación surge del deseo de satisfacer una necesidad y se entiende como todo aquello que mueve e impulsa a una persona a pensar y realizar algo, es una sensación personal de carencia en alguno de los aspectos de la vida de una persona, lo cual genera una desagradable tensión. El individuo se esfuerza por reducir esa tensión, por lo general emprendiendo una acción que le permita satisfacer su necesidad

(Soto, 2001).

El autor sostiene que el emprendimiento se refiere a la motivación al crecimiento; ésta constituye una necesidad que no comprende un balance o equilibrio. 
Una vez lograda, su presencia continúa haciéndose sentir. De hecho, tiende a ser más insaciable en la medida en que lo vamos alimentando.

La motivación comprende aquellos deseos continuos de llenar potencialidades y es la capacidad intrínseca que todo ser siente por dentro para realizar lo que desea.

Es una cuestión de estar auto actualizado. Para Maslow, cuando la persona se conoce y se acepta a sí misma es espontánea, autónoma y creativa. Un individuo emprendedor maduro, sabe de sus posibilidades y limitaciones, es consciente de lo que hace, piensa y siente (Angulo y Santana, 1993). No todos los emprendedores están comprometidos con su vida, muchos se arriesgan sin tener una responsabilidad real, valoran las personas que tienen a su alrededor, disfrutan de lo que hacen y realizan sus acciones sin un norte claro en el horizonte de su empresa. En otras palabras, trabajan con entusiasmo. La espontaneidad es parte de sus acciones y tienen la capacidad para resolver problemas. Les va bien cuando el ambiente en el que se mueven, contribuye a su propio desarrollo, en consecuencia son propicios a las circunstancias del momento. Les va mal, cuando su madurez psicológica es deficiente y su preparación y experiencia en los negocios y la vida no son tomados en serio.

\section{La motivación para David Mc Clelland (1972)}

David McClellan, en su libro The Achieving Society, sostiene que el entrepreneur posee una mayor necesidad del logro o realización que los no emprendedores y que toman riegos moderados.

Desde la perspectiva de Filion (2002), los psicólogos, los psicoanalistas, los sociólogos y otros especialistas del comportamiento humano, son conductistas. Max Weber fue uno de los primeros en interesarse por el tema de los emprendedores, y fue quien en 1930 identificó el sistema de valores como fundamental para explicar su comportamiento, pues los veía como personas innovadoras e independientes que poseían una fuente de autoridad formal debido a su rol de dirigentes en las empresas.

Sin embargo, el mismo autor sostiene que quien le proporcionó el impulso a las ciencias del comportamiento de cara a los emprendedores, fue sin duda, David McClelland. En el transcurso de la década de los cincuenta, se interrogaba sobre el despegue de la Unión de Repúblicas Socialistas Soviéticas y se cuestionaba si el homosovieticus suplantaría al homoamericanus.

McClelland (1961) se asoma a la historia para estudiar el por qué de las grandes civilizaciones e identifica numerosos elementos, entre los que encuentra la presencia de héroes en la literatura. 
Las generaciones siguientes toman a estos héroes como modelos y tienden a imitarlos. Estos héroes tienen la tendencia a saltar las barreras, a agrandar los límites de lo que es posible lograr. Según McClelland, las personas formadas bajo esta influencia desarrollan una fuerte necesidad de realización y dicha necesidad se asocia al emprendedor.

Producto de los acercamientos empíricos de McClelland, el autor estableció rasgos que caracterizan a las personas emprendedoras, asociados particularmente a una alta necesidad de logro e independencia, un alto sentido del control -rasgo estable de la personalidad de los individuos; es la relación entre las acciones que uno realiza y el resultado real alcanzado-, tomadores de riesgos, tolerancia a la ambigüedad y creatividad entre otros.

McClelland (1972), citado por Keith y Newstrom (2003), como teórico destacado sobre las necesidades humanas y la motivación, relaciona el ejercicio empresarial con la motivación de logro, mediante características fundamentales que definen el emprendedor como un individuo que busca el éxito, la competitividad, la aceptación de riesgos moderados, la responsabilidad de sus propias acciones, la constancia, la organización en el trabajo y el planteamiento de objetivos claros y a largo plazo.

Por otra parte, McClelland (1972), afirma que crear empresa tiene que ver con la personalidad emprendedora que crece conforme se agregan motivaciones en el medio junto a las características o atributos personales. Precisamente "esta motivación en conjunto con las habilidades adquiridas y los factores ambientales, llevan al emprendedor a manifestar conductas, por eso el proceso de emprender es dinámico", Barón y Shane (2005).

Las personas son en alguna medida, producto de su medio, deben procurarse ambientes que les permitan ejercitar destrezas, expresar sus actitudes y valores y asumir roles a su agrado.

En resumen, el deseo de éxito lleva al emprendedor a asumir una responsabilidad personal, que permite visualizar las metas, es decir, se ve en la necesidad de mantener estrechas las relaciones interpersonales para socializar sus ideas y buscar la confirmación de su propósito personal.

\section{La conducta humana y los recursos, son un factor de emprendimiento para Howard Stevenson (1990)}

Howard Stevenson, catedrático en el área de iniciativa empresarial de la Universidad de Harvard, define el espíritu emprendedor como el proceso por el cual los individuos persiguen oportunidades sin tener en cuenta los recursos que ellos controlan actualmente (Stevenson \& Jarillo 1990). El emprendedor 
es una persona con características especiales para detectar oportunidades y desarrollarlas, siendo consciente de que no dispone de los recursos necesarios para llevar a cabo su idea de iniciativa. Sin embargo, el emprendedor es capaz de movilizar los recursos ajenos a él para sus fines y aprender a utilizarlos sin buscar la propiedad o pertenencia de dichos bienes. Este comportamiento está condicionado por factores como la imposibilidad de predecir con exactitud los recursos necesarios para lograr la oportunidad; la necesidad de especialización de esos recursos; la necesidad de reducir el riesgo de obsolescencia y de inflexibilidad derivada de la posesión permanente de los recursos, las presiones sociales que exigen un mejor aprovechamiento de los recursos.

\section{El funcionamiento oportunista como conducta del emprendimiento para Gordon Allport (1922)}

La mayoría de los comportamientos humanos están motivados por el funcionamiento como forma expresiva del selfo la participación activa del "yo", lo cual denominó funcionamiento propio, pues la mayoría de las cosas que hacemos en la vida es una cuestión de ser lo que somos. El funcionamiento propio se caracteriza por su tendencia a la actividad, su orientación al futuro siempre visualizado desde el enfoque psicológico.

Los planteamientos de este autor sobre la conducta se centran sobre el concepto de madurez como el elemento fundamental para alcanzar el emprendimiento, lo considera como el mejor progreso para que un individuo funcione dentro de las circunstancias adversas o no de la vida y cuya cualidad sobresaliente está en disponer de la capacidad de proyectar objetivos y el esfuerzo continuo para lograrlos; lo cual es posible a través del aprendizaje intelectual y el raciocinio permanente con su conciencia que es bueno y que es malo.

Allport afirma que existen siete características de salud mental, siempre que la persona disponga de un funcionamiento propio bien desarrollado, a saber:

1. Las extensiones del self basadas en el funcionamiento propio del "yo" duradero y con compromiso.

2. La relación cálida orientada a la dependencia de los demás -la confianza, la empatía, la sinceridad y la tolerancia-.

3. La seguridad emocional y la aceptación propia.

4. La percepción realista.

5. Una medida central de los problemas y desarrollo de las habilidades agrupadas en la solución de problemas.

6. La objetivación del self.

7. La filosofía unificada de la vida. 
La personalidad como fundamento para el emprendimiento de Carl Rogers (1951)

Este autor basa su discurso en el concepto de tendencia actualizante, cuya motivación innata está presente en toda forma de vida dirigida a desarrollar potencialidades hasta el mayor límite posible. Se refiere a la recompensa positiva condicionada de uno mismo, lo que incluye autoestima y una imagen positiva de sí mismo. Afirma que empezamos a querernos si cumplimos con los patrones que otros nos aplican, más que si seguimos nuestra actualización de los potenciales individuales, y destaca como cualidades la apertura a la experiencia, la vivencia existencial, la confianza organísmica, la libertad a la experiencia y la creatividad.

Por otra parte, Carl Rogers considera como lo notable en el del desarrollo de la personalidad, el descubrimiento del yo real, es decir, el conocimiento de sí mismo, basado en la razón y orientado a una vista visión más objetiva del futuro. En este punto, el autor argumenta que las experiencias permiten satisfacer las necesidades personales, y es por esto que la mente se convierte en un campo flexible y abierto a la actuación espontánea, libre franca y creativa, lo que permite entender para dónde va una persona con determinación. Lo anterior, implica crecer y desarrollar en el proceso de experimentación de la vida para alcanzar una mente abierta y un criterio amplio de las cosas.

Como la vida presente es un juego de roles en los que es necesario asumir actuaciones diferentes, porque los retos ya están dados y uno de ellos es el emprendimiento, esta vivencia no se valorará en el individuo mientras no tenga la necesidad y oportunidad de actuar allí. Es la prueba a las potencialidades, a la capacidad de crecer y demostrar que también puede y en la medida que va descubriendo situaciones diferentes por la misma condición de la vida y sus retos, juega a no perder con creatividad.

\section{El liderazgo personalizado en la cultura emprendedora de H. Mintzberg, B. Ahlstrand y J. Lampel (1999)}

La cultura basada en el emprendimiento asocia a la estrategia con un proceso visionario, con énfasis en la formación de una estrategia en el líder único, así como en procesos y estados mentales innatos como la intuición, el criterio, el talento, la capacidad y la percepción. La perspectiva no es colectiva ni cultural, sino personal, 
es la construcción de líder y la organización que responde a los dictados de ese individuo y su personalidad, expresada en la mente del líder. La estrategia emprendedora es premeditada en rasgos generales y de orientación, como emergente en detalles que se adaptan sobre la marcha.

Desde el área del management, se desarrolla la cultura emprendedora basada en un liderazgo personalizado, con visión estratégica como clave para el éxito de la organización. Tanto en negocios como en empresas nuevas. De ahí, J. Pinchot (1985) describe a los intraempresarios -personas con capacidad de desarrollar una idea de negocio en la empresa en la que trabajan- como aquellos que toman iniciativas estratégicas al interior de las organizaciones, en función de un liderazgo personalizado, proactivo y resuelto en las organizaciones, pues

el espíritu emprendedor está muy relacionado con la generación de la visión estratégica y a menudo con el logro de un nuevo concepto. En gran medida, las estrategias se pueden caracterizar como deliberadas, ya que residen en las intenciones del líder. Pero, al ser también en gran parte personales, la visión también puede cambiar; se adapta en el trayecto y está condicionada al aprendizaje sobre la cual pueden surgir nuevas visiones en la medida que el tiempo pasa

(Mintzberg, 1997).

\section{Peter Drucker y la personalidad emprendedora (1964)}

En el planteamiento de Drucker (1964), sobresale el perfil de la personalidad emprendedora; el autor se refiere a ser innovador como la capacidad que tiene una persona para determinar los recursos y canalizar los esfuerzos a fin de aprovechar las oportunidades, la práctica basada en conocimientos previos, la personalidad emprendedora y la capacidad de trabajo con personas inflexibles y pragmáticas. Veamos desarrollos de los anteriores argumentos de Drucker.

El trabajo emprendedor requiere formas innovadoras de conseguir nuevos productos y servicios. $\mathrm{Y}$ de obtener un gran conocimiento de sí mismo, del mercado y de sus empleados. Se debe tener en cuenta la atención a la retroalimentación en términos de costo-beneficio. El empresario innovador se basa en la teoría económica y social que contempla el cambio como algo normal y saludable, y considera que la tarea más importante de la sociedad, especialmente de la economía, es hacer algo diferente (Drucker, 1986).

Drucker (1964) afirma que el trabajo del emprendedor es determinar los recursos y los esfuerzos para maximizar oportunidades más que beneficios, buscar la eficacia más que la eficiencia; e indaga cómo encontrar qué se debe hacer, más que cómo se debe hacer, o bien, 
asignar recursos a las oportunidades antes que a los problemas.

Entiéndase el empresariado y el espíritu emprendedor no como una ciencia, ni como un rasgo de personalidad del empresario, sino como una práctica basada en conocimientos teóricos en las acciones y las conductas de los empresarios.

La personalidad misma de emprendedor se basa en la toma decisiones, visión e intuición del individuo. Es preciso sintetizar que, para este autor, el emprendedor exitoso comprende dos rasgos de conducta: el de emprendimiento y el de administración.

Mientras que la conducta administrativa se cuestiona ¿dónde está la oportunidad apropiada?, ¿qué recursos controlo?, ¿cómo minimizo el impacto de los demás para poder operar?, ¿qué estructura determina la relación entre la empresa y el mercado?, ¿qué acciones puedo desarrollar en este entorno?

La conducta emprendedora trabaja con personas inflexibles y pragmáticas; impulsadas por la necesidad de logro; con educación formal e informal; un alto sentido de independencia desde su primera infancia, pues se enfrentaron a situaciones difíciles en los primeros años de vida; gran necesidad de control; sentimientos de inseguridad y temor por no alcanzar la seguridad; resentimiento ante la autoridad; $y$ tendencia a aceptar riesgos moderados.
La personalidad centrada en un ambiente vocacional Holland J. L. (2001)

Este autor plantea que cada persona proyecta sobre las ocupaciones sus puntos de vista acerca de sí misma y del ambiente laboral que prefiere. Para ello utiliza estereotipos vocacionales, es decir, un juicio formulado con respecto a alguien, según el criterio de la percepción propia del grupo al cual esa persona pertenece, (Soto, 2001).

Las personas eligen una actividad, pues la imagen que tienen de ella, por lo general un estereotipo, les parece atractiva y se identifican con ella.

Los individuos de una misma vocación tienen personalidades parecidas e historias similares de desarrollo. Por este motivo responderán de la misma manera frente a diversas situaciones y crearán ambientes interpersonales característicos. Esta congruencia entre la personalidad y el ambiente académico, laboral o profesional, determina el grado de satisfacción de estabilidad y del logro de la elección vocacional.

Holland (2001) determinó que la personalidad se manifiesta en varios prototipos de individuos, por eso los clasifica así:

- El realista que prefiere las actividades en las que se requieren de habilidad y fuerza, porque es conformista y práctico. 
- El prototipo convencional que se activa frente a lo reglamentado, ordenado y definido, porque es resignado y poco imaginativo.

- El tipo investigador, encaminado a actividades en las que se abstrae pensando, constituyendo y comprendiendo, y no actúa porque su interior lo conduce a ser analítico.

- El original, curioso e independiente.

- El prototipo social cuya actividad está enfocada a servir a otros; esto le produce satisfacción personal, formar y ayudar es su tarea, de ahí que sea sociable, amigable, cooperador, comprensivo y solidario.

- El prototipo artístico, centrado en actividades confusas y poco preparadas, en las que la espontaneidad juega un papel fundamental, y la improvisación es imaginativa, desordenada, emotiva y con pensamiento lateral y poco práctico.

- El prototipo emprendedor como una persona ambiciosa, enérgica y dominante, que prefiere actividades en las que use la expresión verbal, trata de influir en los demás y, en la medida en que avanza, va utilizando el poder. Su satisfacción se encuentra en las relaciones públicas al conocer, tener contacto con otras personas e interactuar con ellas.

\section{Las necesidades humanas fundamentales Manfred Max Neef (1999)}

Las necesidades no sólo se consideran son carencias, sino potencialidades del ser humano, desde el punto de vista ontológico, que se manifiestan en ser, estar, tener y hacer; sin embargo, desde el punto de vista axiológico -valores humanos-, estas variables se encuentran en la subsistencia, la protección, el afecto, el entendimiento, la participación, el ocio, la creación, la identidad y la libertad. Dichas necesidades se suplen o se inhiben a través de agentes, que representan las distintas formas de necesidad. Cultiva y planea sus acciones teniendo en cuenta sus actitudes desde los diversos agentes que potencian la calidad de vida de las familias.

\section{El aprendizaje es la razón del emprendimiento para Andy Freire (2005)}

Para el emprendedor nato todo es exitoso, Freire (2005) afirma que todo se hace, que nada es innato y que todo se puede aprender y desarrollar. Existe una capa de hábitos técnicos fácilmente modificables, éstos tienen que ver con la formación y el aprendizaje educativo, la formación universitaria, las interacciones con mentores experimentados, los empresarios 
consumados, los estudiosos de una actividad específica para aprender cómo funciona lo que se desea poner a funcionar. En razón a lo anterior, las universidades del mundo tratan de enseñarlo y practicarlo como una salida a las escasas posibilidades de empleo que ofrecen los Estados y las crisis marginales laborales de las empresas.

Otra parte del emprendedor, es la capa neuronal inmodificable, por ser innata y atender a causas genéticas, la cual presenta una herencia condicionada propia de un entorno familiar, y cuyas habilidades provienen de la misma herencia; $y$ es inimaginable, por mucho empeño que le ponga, que alguien adquiera la capacidad a voluntad propia de hacer empresa.

Entre lo claramente modificable y lo inmodificable, está el área gris de lo modificable, a esto se le denomina meta-habilidades -competencias básicas del individuo que tienen que ver con los procesos de inculturación-. Si el emprendedor tiene el compromiso firme para mejorarlas o desarrollarlas, no le bastará aprender la dimensión técnica. Esta es necesaria, pero no suficiente; con ella será un emprendedor más preparado en lo técnico, para ello deberá desarrollar esas meta-habilidades o competencias para asumir el papel de protagonista continuamente dispuesto a aprender y a desarrollar su autoestima para lograr mayor firmeza en sus decisiones, motivarse por la idea de emprender, compartir con un equipo, asumir riesgos para lograr su independencia y divertirse en el proceso con aciertos y errores (Freire, 2005).

\section{Algunas reflexiones finales}

\section{Los límites explicativos del enfoque psicológico ohumanistaalainvención} y creatividad de los emprendedores

Aún están pendientes algunos desarrollos investigativos desde la escuela psicológica, sobre la invención y la creatividad de los emprendedores, como característica aceptada y asociada a éstos. Según Wompner (2005), "los emprendedores se caracterizan porque inventan, adaptan, reconocen e impulsan nuevas ideas hasta trasformarlas en nuevas oportunidades", es decir, poseen creatividad, pero "la psicología moderna está todavía lejos de poder explicar la creatividad en términos lógicos y objetivos, pero en años recientes se han hecho adelantos en cuanto a la comprensión de los tipos de personalidad creativa y de las circunstancias en que es más fácil que aparezcan”.

\section{La complejidad del emprendimien- to supera la simple pregunta ¿nacen o se hacen?}

A pesar de los importantes desarrollos y aportes al emprendimiento por parte de la escuela psicológica, aún persiste y persistirá por mucho tiempo, la pregunta ¿los emprendedores nacen o se hacen? 
$\mathrm{Al}$ interior de la escuela ha surgido esta pregunta, hasta ahora sin respuesta, pero con dos corrientes, una sustentando que nacen y otra argumentando que se hacen. En la primera corriente, se encuentran Alan Jacobwitz, con las características personales de los emprendedores: incansables, independientes, tendencia a estar solos, y una extrema autoestima; otros, como Schein, 1994 y Solomon 1989, además han incluido características como innovadores, orientados a la acción, alta necesidad de control personal y autonomía. En la segunda corriente, están Kreuger y Brazeal (1994) quienes ofrecen un modelo dinámico que sugiere que la intención emprendedora está basada en la interacción entre las características personales, percepciones, valores, creencias, pasado y entorno -contexto situacional-. Los autores basan esta afirmación en el modelo de Shapero, del evento emprendedor, en el que entrepreneurship se define como "la caza de la oportunidad sin tener en cuenta los procesos existentes".

(Kreuger \& Brazeal, 1994).

Tras un riguroso estudio que buscaba definir las características personales de los emprendedores y sus diferencias con los ejecutivos corporativos, basado en un cuestionario que incluía ocho tipos de preguntas, para un total de diecinueve, aplicadas a veintidós individuos de ambos sexos; los autores determinaron que los mismos emprendedores y los temas del entrepreneurship son más complejos que estas opciones", y sostienen que "se considera que los emprendedores poseen determinadas características particulares que se manifiestan desde edad temprana. De esta manera la enseñanza del entrepreneurship podrá lograr nuevos y mejores emprendedores entre quienes posean las aptitudes".

Complejizar el emprendimiento de manera multidimensional

Como lo argumenta Porras (2006), en el emprendimiento, se reconocen tres enfoques, desde la perspectiva de la empresarialidad o sobre la creación de empresas:

- Desde la ciencias humanas o la escuela psicológica.

- Desde una tendencia economicista.

- Desde las ciencias de la gestión y la organización (Fayole y Bruyat, 2002).

Es decir, el fenómeno se aborda de forma fragmentada y requiere ser planteado en nuevas perspectivas integradoras, complejas y multidimensionales, pues según Filion (2002), observamos con Mulholland (1994), Rosa y Bowes (1990), que el campo de estudio y análisis está dominado por los positivistas-funcionalistas y que existe una gran necesidad de abrir aún más las nuevas perspectivas para comprender mejor el conjunto de lo que es y de lo que hace el emprendedor. 


\section{Referencias}

1. Аmit, R. \& Muller, E. (1994). Contrasting Attributes and Attitudes on Entrepreneurs and non-Entrepreneurs Profound. The Journal of Management Studies, No. 5 (33).

2. Angulo, M.H. y Santana L. (1993, mayo-diciembre). El desarrollo de la personalidad emprendedora: un programa de formación empresarial. Revista EAN: Escuela de Administración de Negocios, Núm. 19, pp. 34-46.

3. Aragón, F. y Severi, M. (2001). Características personales de los emprendedores: diferencias con los ejecutivos de corporaciones. Tesis de maestría no publicada, Universidad del cema. Buenos Aires, Argentina.

4. Castillo, A. (1999). Estado del arte en la enseñanza del emprendimiento. Chile: INTEC.

5. Drucker, P. (1964). Managing for Results. New York: Harper \& Row. 6. (1986). The Frontiers of Management. England: First plume Printer.

7. . (2005). Innovation and Entrepreneurship: Practice and Principles. Great Britain: Editorial вн.

8. Davis, K. \& Newstrom, J. (2003). Comportamiento Humano en el trabajo: comportamiento organizacional. (11 ${ }^{\text {a }}$ Ed.). México, Mc Graw Hill.

9. Fayolle A. y Bruyat C. (2002, noviembre 21 - 22). A conceptual and methodological framework to study, using the modelling of complex system, the foundation and the development procesess of innovative bussiness activities. RENT $X V I$, Workshop conference proceedings. Barcelona, España.

10 Filion, L. (2002, julio). Emprendedores y pequeños propietarios de la pequeña-mediana empresa: PME. Administración y organizaciones, pp. 113-152.

11. Freire, A. (2005). Pasión por emprender: de la idea a la cruda realidad. Bogotá: Norma.

12. GonzÁlez, J. (2003). Comprensiones de la iniciativa emprendedora desde su historia, el individuo y el proceso. Estado del arte. Recuperado el 16 de septiembre de 2009, desde http://www.emprendimientolocalyregional.com/blogs/index. php?blog=11.

13. Holland, J. (2001). Corporate Value Creation. Glasgow: University of Glasgow.

14. Krause, M. \& Thomas, L. (2004). On-the-Job Search and the Cyclical Dynamics of the Labor Market. Economics Working Paper. The Johns Hopkins University, Department of Economics, Archive 513.

15. Krueger, N.; Brazael, D.V. (1994). Entreprenurial Potential \& Potential Entrepreneurs. Entrepreneurship Theory and Practice, pp. 91- 104. 
16. Lachman, R. (1980). Toward Measurement of Entrepreneurial Tendencies. Magnament International Revierw, No. 20 (2), pp. 108- 116.

17. Lucas, J. (2004). El concepto de "espiritu emprendedor" y su evolución bistórica. Documento de trabajo uca, Núm. 2, pp. 1-20.

18. Mulholland, R. (1994, octubre 27 al 29). Approaches to Entrepreneurship Research. En Mount, J. (Ed.), Proceedings of the $11^{\text {th }}$ Annual Conference Canadian Council for Small Business and Entrepreneurship, Winnipeg. pp. 122-133.

19. Mintzberg, H., Brian J. y Voyer, J. (1997). El proceso estratégico. México: Prentice Hall.

20. Рinchot, G. (1985) Intrapreneuring: Why you don't to Leave the Corporation to Become an entrepreneur. Cambridge: Harper \& Row.

21. Porras, J. (2006). Diseño conceptual del emprendimiento para el desarrollo regional desde la complejidad autopoiésica. Maestría en Administración. Universidad Nacional de Colombia.
22. Rogers, C. (1951). El proceso de convertirse en persona. Buenos Aires: Paidos.

23. Rosa, P.y Bowes, A. (1990). La iniciativa empresarial: algunas lecciones de antropología social. E.C.S.B. 4th Workshop on research in entrepreneurship, University of Cologne, Alemania.

24. Scheinberg, D. \& McMillan, J. (1988). An Eleven Country Study of the Motivations to Start a Business. Englewood Cliffs, N.J.: Prentice Hall.

25. Soто, E. (2001). Comportamiento organizacional. México: Editorial Thompson Learnig.

26. Stevenson, H.H. \& Jarillo, J.C. (1990). A Paradigm of Entrepreneurship: Entrepreneurial Management. Strategic Management Journal. No. 11, pp. 17-27.

27. Villa, M. (2008). El espíritu emprendedor, la gran fuerza del progreso. Cuadernos de pensamiento político, Núm.18, pp. 215-232.

28. Wompner, F. (2005). Educación superior para el emprendimiento. Serie Indagación, Expansiva. Núm. 19, pp. 1-16. 